\title{
Two-Tier Hierarchical Cyber-Physical Security Analysis Framework For Smart Grid
}

\author{
Jin Wei and Deepa Kundur \\ Department of Electrical and Computer Engineering \\ Texas A\&M University, College Station, TX 77843, USA
}

\begin{abstract}
We propose a two-tier hierarchical cyber-physical framework for analyzing transient stability in the smart grid in the face of cyber-physical attack. We model the smart grid as a networked multi-agent dynamical system in which each agent includes multiple generators, measurement devices and local control. The agents interact with each other through cyberphysical integrated coordination and the generators within the same agent interact through the physical couplings.

We present a cyber-physical flocking-based protocol to address the issue of system faults. We extend this work to derive a spectral matrix based generator coherency identification algorithm to analyze the coherency amongst synchronized generators after fault clearing. Generators with high coherency are grouped in an agent while those with low coherency form another. For each agent, the generator having highest inertia is selected as the lead component.

In order to reduce the required communication and energy of the control protocol, cyber interaction between agents is realized by coupling only lead generators. By using our developed flocking-based protocol, all the lead generators achieve consensus to the desired nominal frequency and their phase angles achieve phase cohesiveness. Subsequently secondary generators within each agent are able track these stabilizing lead dynamics due to strong physical couplings. Simulation results are presented to support the proposed framework.
\end{abstract}

\section{INTRODUCTION}

It is well known that the smart grid promises increased reliability, efficiency and security through the use of advanced energy- and information-related infrastructure. Modeling of such system interaction is a critical part of system design, development and implementation. Thus, much recent research activity has focused on challenges of cyber-physical system modeling.

In this work, we present a framework for analyzing issues involving transient stability within a smart grid. Accordingly, our models take on a cyber-physical flavor representing both physical couplings and information-related control. We focus on the problem of achieving transient stability in the presence of a system fault (a consequence of cyber and/or physical disruption) using distributed control. In particular, we consider a scenario in which active control is computed using local and distributed PMU data and employs short-time power injection/absorption at key generator buses via smart grid-enabled fast-reacting sources/sinks such as batteries and flywheels.

In this paper, we extend our prior work [1], [2] and leverage physical coherence amongst generators to reduce energy and communications related costs for control. Our approach identifies coherent generator groups to enable derivation of a lower-cost control law that effectively leverages the physical coupling amongst such clusters to achieve stability with lower overhead.

Our flocking-based control paradigm ensures the overall cyber-physical system has the objectives of exponential frequency synchronization and phase cohesiveness through biologically inspired strategies used for flocking such as consensus, navigation and steering. In this paper, we demonstrate how this framework conveniently lends itself to spectral bisection of the physical coupling matrices to identify coherent generator groups. This in turn leads to a strategy of selective cyber control at only "lead" generators that we describe.

Section II reviews our flocking-based cyber-physical framework. Our proposed approach to coherency identification of generators is presented in Section III and is illustrated via the WECC 3-machine 9-bus system. The two-tier hierarchical control framework is described in Section IV followed by final remarks in Section V.

\section{Flocking-BASED CYBER-PHYSICAL FRAMEWORK}

The derivation of our cyber-physical smart grid model is detailed in [1], [2]. Here, we provide a brief overview of our framework. Our construction begins with a smart grid topology and aims to create a scalable depiction relating both physical and cyber components. To address issues of transient stability, these mathematical descriptions relate generator rotor angles and frequencies. Thus, our construction employs the classical swing equation model of a synchronous generator and considers a Kron-reduced power system. The following well-known equations result for an $N$-generator system:

$M_{i} \dot{\omega}_{i}=-D_{i} \omega_{i}+P_{m, i}-\left|E_{i}\right|^{2} G_{i i}-\sum_{j=1}^{N} P_{i j} \sin \left(\theta_{i}-\theta_{j}+\varphi_{i j}\right)$,

where $i \in\{1,2, \ldots, N\}$ represents the generator index, $\theta_{i}$ denotes the rotor phase angle measured with respect to a rotating frame reference at frequency $f_{0}=60 \mathrm{~Hz}, \omega_{i}=\dot{\theta}_{i}$ is the relative normalized frequency, $M_{i}>0$ and $D_{i}>0$ represent the generator inertia and the damping parameters, respectively, $E_{i}, P_{m, i}$ and $G_{i i}$ are the internal voltage, mechanical power input and equivalent shunt conductance of Generator $i$, respectively. $P_{i j}=\left|E_{i}\right|\left|E_{j}\right|\left|Y_{i j}\right|$ and $\varphi_{i j}=$ $\arctan \left(G_{i j} / B_{i j}\right)$ where $Y_{i j}, G_{i j}$ and $B_{i j}$ are the Kronreduced equivalent admittance, conductance and susceptance, respectively, between Generators $i$ and $j$. 
We integrate the influence of the cyber network through an active control strategy in which power injection/absorption is employed at each generator bus to aid in transient stabilization in the face of physical disruption. We assume that a fastreacting power source $P_{u, i}$ facilitated through smart grid technology such as flywheels or battery storage is available and its associated control signal $u_{i}=P_{u, i}$ is computed by employing sensor measurements available through the cyber network. The cyber-physical coupling can therefore be represented as:

$$
\begin{aligned}
M_{i} \dot{\omega}_{i}= & -D_{i} \omega_{i}+P_{m, i}+\underbrace{u_{i}}_{\text {cyber influence }}-\left|E_{i}\right|^{2} G_{i i} \\
& -\sum_{j=1}^{N} P_{i j} \sin \left(\theta_{i}-\theta_{j}+\varphi_{i j}\right) .
\end{aligned}
$$

Eq. (2) carries the flavor of dynamical system models of flocking behavior [3]. Thus, inspired by the analogies present between the requirements for transient stability and flocking rules in nature, we develop a flocking-based control protocol that consists of four terms: a gradient-based term, consensus term, navigation feedback and a component designed to enable singular perturbation analysis. The proposed cyber-physical system can be described as [1], [2]:

$$
\left\{\begin{array}{l}
\dot{\boldsymbol{\theta}}=\boldsymbol{\omega} \\
\mathbf{M} \dot{\boldsymbol{\omega}}=\widehat{\mathbf{u}}=-\nabla \mathbf{V}-\mathbf{L} \boldsymbol{\omega}-\mathbf{G} \boldsymbol{\omega}-c_{1}\left(\boldsymbol{\omega}-\boldsymbol{\omega}^{*}\right)
\end{array}\right.
$$

where $\boldsymbol{\theta}=\left[\theta_{1}, \ldots, \theta_{N}\right]^{T}, \boldsymbol{\omega}=\left[\omega_{1}, \ldots, \omega_{N}\right]^{T}, \mathbf{M}=\mathbf{D}(\mathbf{I}+\mathbf{B})$, $\mathbf{I}$ is the identity matrix, $\mathbf{D}=\operatorname{diag}\left[D_{1}, \ldots, D_{N}\right], \mathbf{B}$ is a $N \times$ $N$ cyber coupling matrix designed to relax the over-damped generator assumption, $\mathbf{L}$ is the physical coupling matrix which is determined by the impedances of the Kron-reduced power system topology, $\mathbf{G}$ is another $N \times N$ cyber coupling matrix designed to achieve frequency consensus, $\nabla \mathbf{V}$ is the gradientbased term designed to ensure the the limited generator phase angle differences of $\frac{5 \pi}{9}, c_{1}$ is the parameter for the linear navigational feedback and $\boldsymbol{\omega}^{*}=\mathbf{0}$ is the desired normalized generator frequency.

The flocking-based active control strategy requires power injection/absorption at each generator bus, but facilitates transient stability and increased critical clearing time. We next describe an approach to leverage the physical coupling amongst generators to apply such control only to "lead" generator buses thus saving control cost while maintaining its advantages.

\section{Spectral Matrix-Based Generator Coherency IDENTIFICATION}

To identify generator coherency, we make use of insights recently presented by Dörfler and Bullo in [4] whereby it is shown via singular perturbation analysis that Eq. (1) is equivalent to:

$$
D_{i} \omega_{i}=P_{m, i}-\left|E_{i}\right|^{2} G_{i i}-\sum_{j=1}^{N} P_{i j} \sin \left(\theta_{i}-\theta_{j}+\varphi_{i j}\right) .
$$

Taking the derivative of both sides, Eq. (4) can be compactly represented as [4]:

$$
\mathbf{D} \dot{\omega}=-\mathbf{L} \boldsymbol{\omega}
$$

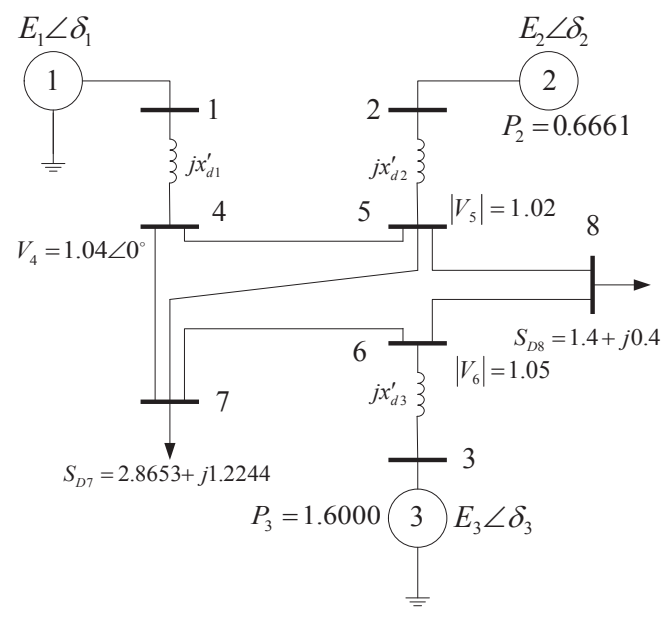

Fig. 1. WECC 9-bus power system.

where $\mathbf{D}=\operatorname{diag}\left[D_{1}, D_{2}, \ldots, D_{N}\right], \boldsymbol{\omega}=\left[\omega_{1}, \ldots, \omega_{N}\right]^{T}, \mathbf{L}$ is a $N \times N$ matrix whose elements $l_{i j}$ are defined as:

$$
l_{i j}= \begin{cases}-P_{i j} \cos \left(\theta_{i}-\theta_{j}+\varphi_{i j}\right), & \text { if } i \neq j . \\ \sum_{k=1}^{N-1} P_{i k} \cos \left(\theta_{i}-\theta_{k}+\varphi_{i k}\right), & \text { otherwise. }\end{cases}
$$

It can be shown that the matrix $\mathbf{L}$ can be interpreted as the Laplacian (with zero row-sum and positive semi-definite (PSD) character) of a directed weighted graph $\mathcal{G}$ associated with the power system topology for a small time interval after the fault is cleared; the associated weight of an edge $e_{i j}$ in $\mathcal{G}$ would be given by $l_{i j}$.

It is well known that for Laplacian matrices, the second smallest eigenvalue $\lambda_{2}$ represents the algebraic connectivity of its associated graph. Moreover, for our framework the signs of the elements of the associated eigenvector $\mathbf{v}$ (called the Fedler vector) provide information for spectral bisection [5], [6] to partition $\mathcal{G}$ into two relatively disjoint (in terms of physical coupling) subgraphs. In the case of bisection, one group of generators will represent high coherency and the other low.

Our generator coherency identification algorithm is summarized in Table I. We assume that the number of generator groups to partition is known a priori and denoted $\vartheta$. Please note that the algorithm is given for $\vartheta$ a power of two, but more general values of coherency groups can be considered.

The algorithm is designed to be applied shortly after a system fault is cleared to analyze coherency just prior to the application of control. Thus, our approach is fault-dependent and real-time. After identification of coherent clusters, the twotier hierarchical controlling framework proposed in Section IV is applied.

We next illustrate our proposed spectral matrix-based generator coherency identification method on the WECC 3-machine 9-bus power system of Fig. 1 through two case studies employing SimPowerSystems.

\section{A. Case I}

In the first case, a 3-phase short circuit is applied to Bus 6 at $t=0$ and then is cleared at $t=0.3 \mathrm{~s}$ by removing Line 
Table I

Spectral Matrix BASEd Generator CoHerency IDENTIFICATION METHOD

Given a predetermined number of the generator groups $\vartheta=2^{C}$.

1. Obtain the Laplacian matrix $\mathbf{L}$ by using Eq. (6).

2. Calculate the eigenvector $\mathbf{v}$ corresponding to the smallest nontrivial eigenvalue of the laplacian matrix.

3. For $k_{1}=1: N$

if the $k$ th element of the eigenvector $\mathbf{v}\left(k_{1}\right)>0$ else the $k$ th generator belonging to the partition $\mathcal{S}_{+}$,

End the $k_{1}$ th generator belonging to the partition $\mathcal{S}_{-}$,

4. If $C>1$

for $k_{2}=2: C$

1). Remove the edges connecting the two partitions $\mathcal{S}_{+}$and $\mathcal{S}_{-}$from the graph $\mathcal{G}$ and achieve two disconnected graphs $\mathcal{G}_{+}$and $\mathcal{G}_{-}$associated with $\mathcal{S}_{+}$and $\mathcal{S}_{-}$, respectively.

2). For $\mathcal{G}_{+}$and $\mathcal{G}_{-}$, respectively, achieve the associated end Laplacian matrix $\mathbf{L}_{+}$and $\mathbf{L}_{-}$and repeat the steps 2 and 3.

Else End

$6-7$. The plots of the generators' frequencies and the phase angles are shown in Fig. 2. As observed from Fig. 2, the phase angles of Generators $G_{1}$ and $G_{2}$ are coherent in contrast to $G_{3}$.

To demonstrate our approach, we assume that at $t=0.4 \mathrm{~s}$, the proposed generator coherency identification algorithm is applied. The predetermined number of generator groups is $\vartheta=$ 2 thus spectral bisection is applied only once. At $t=0.4 \mathrm{~s}$, the Laplacian matrix $\mathbf{L}$, eigenvalue $\lambda_{2}$ and associated Fedler vector $\mathbf{v}$ are given by:

$$
\begin{aligned}
\mathbf{L} & =\left(\begin{array}{ccc}
2.4465 & -1.8037 & -0.6428 \\
-1.8068 & 2.2250 & -0.4182 \\
-0.6184 & -0.3979 & 1.0164
\end{array}\right), \\
\lambda_{2} & =1.5327 \\
\mathbf{v} & =[-0.3663,-0.4715,0.8022]^{T} .
\end{aligned}
$$

Based on the signs of the elements of the eigenvector $\mathbf{v}$, we conclude that the $\vartheta=2$ classes of coherent generators are given by: $\left\{G_{1}, G_{2}\right\}$ and $\left\{G_{3}\right\}$ as previously observed in Fig. 2 .

\section{B. Case II}

In the second study, a 3-phase short is applied to the middle of Line $4-5$ and then is cleared once again at $t=0.3 \mathrm{~s}$ by removing that line. Generator frequencies and phase angles are shown in Fig. 3. This time, coherence between Generators $G_{2}$ and $G_{3}$ is observed.

The proposed coherence identification approach is applied at $t=0.4 \mathrm{~s}$ and for $\vartheta=2$ to provide the following values for $\mathbf{L}, \mathbf{L}$ and $\mathbf{v}$ :

$$
\begin{aligned}
\mathbf{L} & =\left(\begin{array}{ccc}
1.0129 & -0.4633 & -0.5496 \\
-0.4816 & 1.6161 & -1.1344 \\
-0.5703 & -1.1329 & 1.7032
\end{array}\right), \\
\lambda_{2} & =1.5343 \\
\mathbf{v} & =[-0.8045,0.4608,0.3747]^{T} .
\end{aligned}
$$

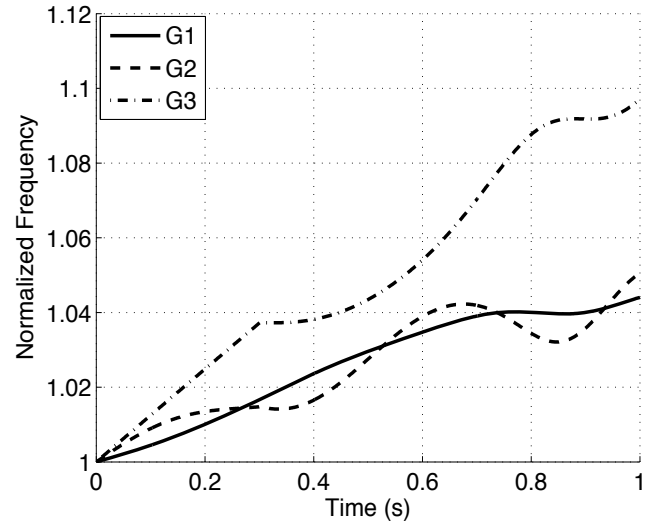

(a)

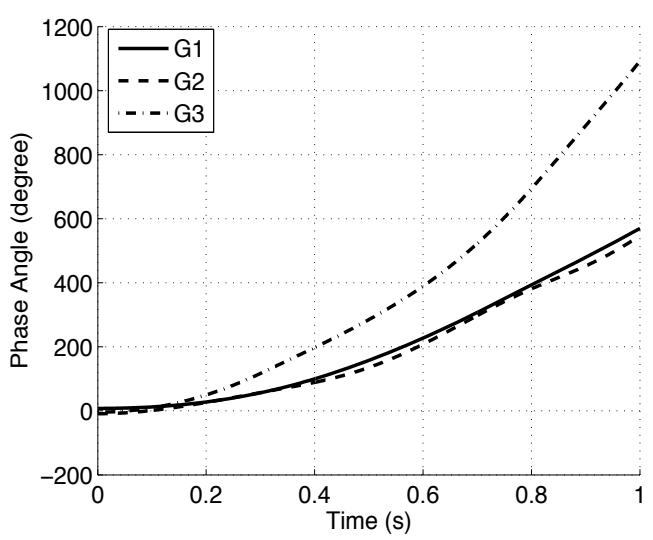

(b)

Fig. 2. 3-phase short at Bus 6. (a) Normalized frequencies, (b) Phase angles.

Similarly, given $\mathbf{v}$, we conclude that $\vartheta=2$ classes of coherent generators are given by: $\left\{G_{1}\right\}$ and $\left\{G_{2}, G_{3}\right\}$ as verified in Fig. 3.

\section{Two-TIER HierarchicAl CONTROL FramewORK}

Scalability of our flocking-based approach for transient stability control is facilitated through exploiting physical couplings amongst generators. Specifically, we propose a twotier hierarchical control framework in which a lead generator is identified within each coherent group such that control is applied only at the lead generator buses; the generator having highest inertia is selected as the lead component. This reduces both energy and communication overhead. The active control guarantees transient stability of the lead generators (tier-1) and the physical couplings facilitate overall consensus of the secondary generator frequency states (tier-2). As a result all generator frequencies converge to the reference frequency (i.e., typically $60 \mathrm{~Hz}$ ) and phase cohesiveness is established. Fig. 4 illustrates our framework for the WECC 3-machine 9-bus system for Case I coherence.

Under this tiered framework, the complexity of the overall cyber control protocol is reduced and the cyber control law is established as presented in Eq. 3 and in [2] except that it is 


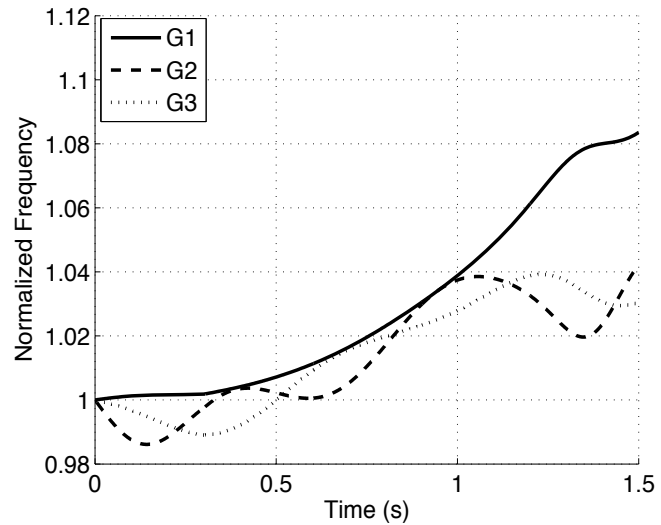

(a)

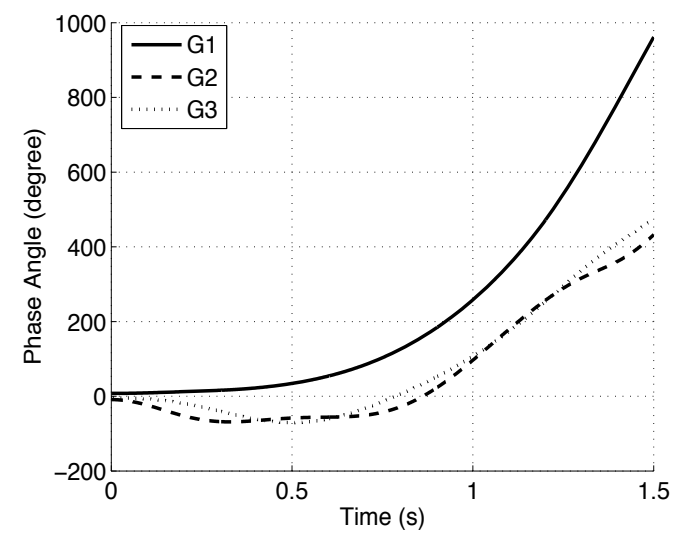

(b)

Fig. 3. 3-phase short at Line 4-5. (a) Norm. frequencies, (b) Phase angles. applied to the reference graph $\mathcal{G}$ of the two-tier system, created by taking the original graph of the Kron-reduced system and eliminating cyber links of non-lead generators. Similarly, the Laplacian matrix $\widetilde{\mathbf{L}}$ used for control computation is generated by excluding rows and columns from the original system Laplacian $\mathbf{L}$ of Eq. 5 that do not correspond to lead generators.

Figs. 5 and 6 demonstrate the performance of the twotier controller to provide transient stability in the face of the faults discussed for Case I and II, respectively. In both cases, the control is activated at time $t=0.4 \mathrm{~s}$ and a maximum transmission capacity is assumed for the fast reacting grid thus limiting the magnitude of $P_{u, i}$ by $P_{u, i} / P_{m, i} \leq 1$ as illustrated in Fig. 5(d). In Case I, $G_{1}$ and $G_{3}$ are selected as lead generators and the navigational feedback parameter is set to $c_{1}=3$. In Case II, $G_{1}$ and $G_{2}$ are leads and $c_{1}=1$.

\section{Final Remarks and Future Work}

In this paper, we have introduced a two-tier hierarchical cyber-physical analysis framework for analyzing attacks and mitigation approaches in smart grid systems. The framework has great potential for identifying system level design strategies to address issues of cyber-physical security.

Our hierarchical approach enables the grouping of coherent generators into agents and leverages their physical couplings for intra-agent generator transient stabilization. Inter-agent stabilization is facilitated through cyber control applied to lead generators of each agent. Our insights on coherency identification enable more efficient control to be applied. Future work will focus on employing the information provided by our approach to identify generator clusters to design optimal strategies of cyber-physical attack to leverage physical couplings for cascading failures.

\section{ACKNOWLEDGEMENTS}

Research funding was provided by the U.S. National Science Foundation under grant ECCS-1028246 and the Norman Hackerman Advanced Research Program Project Number 000512-0111-2009.

\section{REFERENCES}

[1] J. Wei, D. Kundur, T. Zourntos, and K. L. Butler-Purry, "A secure cyberphysical integration framework for wide-area power systems under communication delay," in Proc. International Conference on High Confidence Networked Systems (HiCoNS), CPSWeek 2012, Beijing, China, April 2012 , p. under review.

[2] J. Wei, D. Kundur, T. Zourntos, and K. Butler-Purry, "A flocking-based dynamical systems paradigm for smart power system analysis," in Proc. IEEE Power Engineering Society General Meeting, San Diego, CA, July 2012, p. under review.

[3] R. Olfati-Saber, J. Fax, and R. Murray, "Consensus and cooperation in networked multi-agent systems," Proceedings of the IEEE, vol. 95, no. 1, pp. 215-233, January 2007.

[4] F. Dörfler and F. Bullo, "Topological equivalence of a structure-preserving power network model and a non-uniform Kuramoto model of coupled oscillators," in Proc. IEEE Conference on Decision and Control and European Control Conference, Orlando, FL, December 2011, pp. 70997104.

[5] M. Fiedler, "Algebraic connectivity of graphs," Czechoslovak Mathematical Journal, vol. 23, pp. 298-305, 1973.

[6] A. Seary and W. Richards, Dynamic Social Network Modeling and Analysis. National Academies' Press, 2003. 


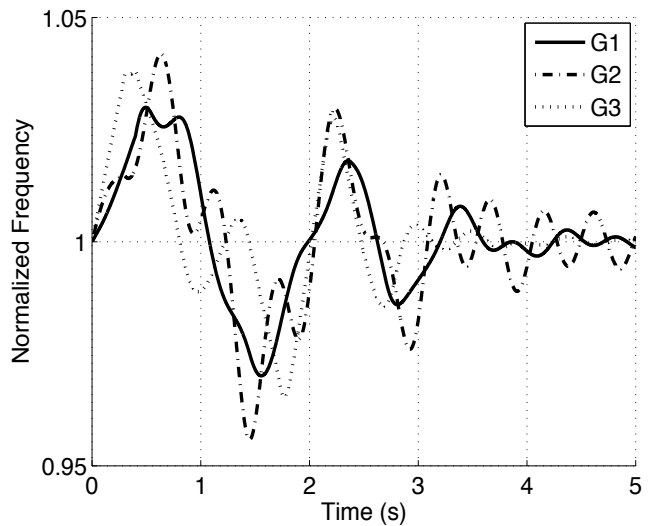

(a)

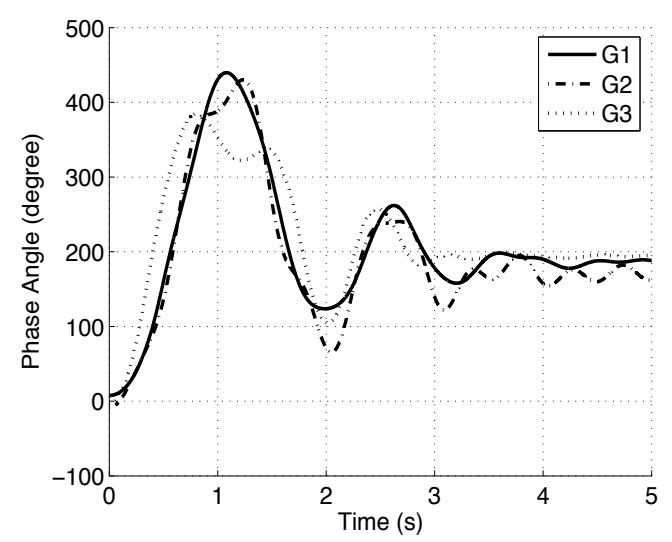

(b)

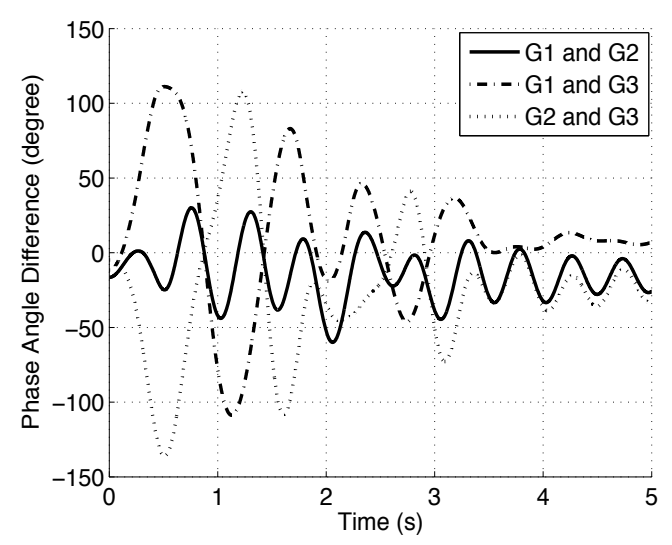

(c)

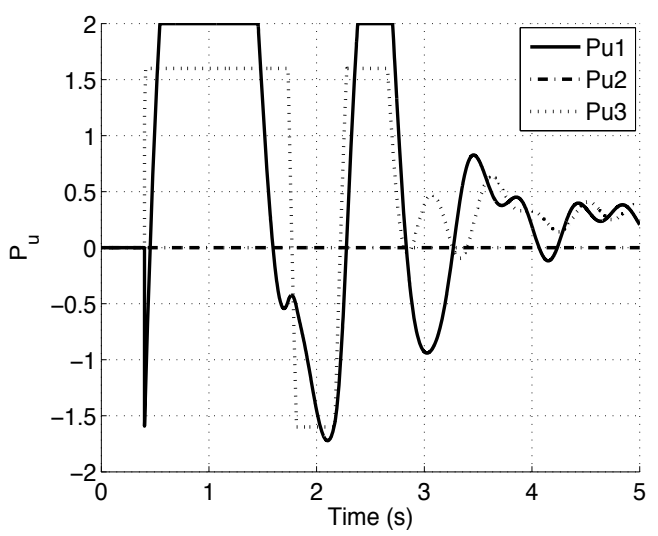

(d)

Fig. 5. Results of Two-Tier Control for Case I.

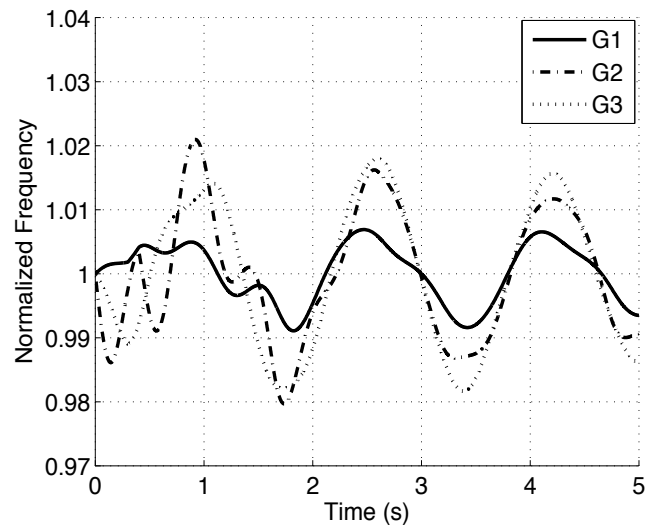

(a)

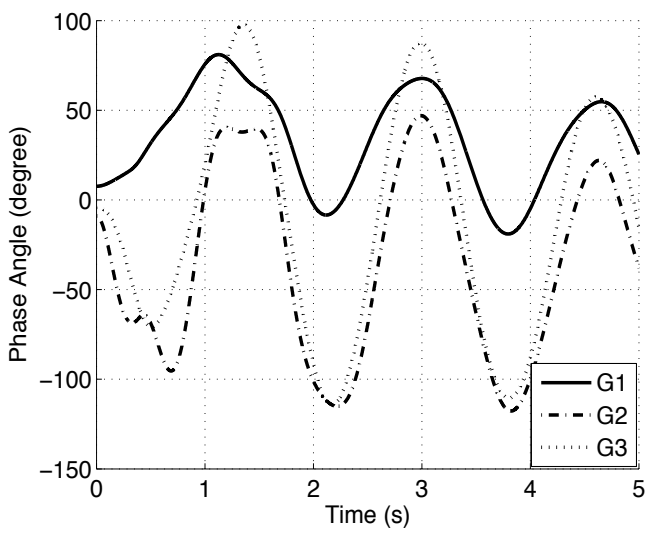

(b)

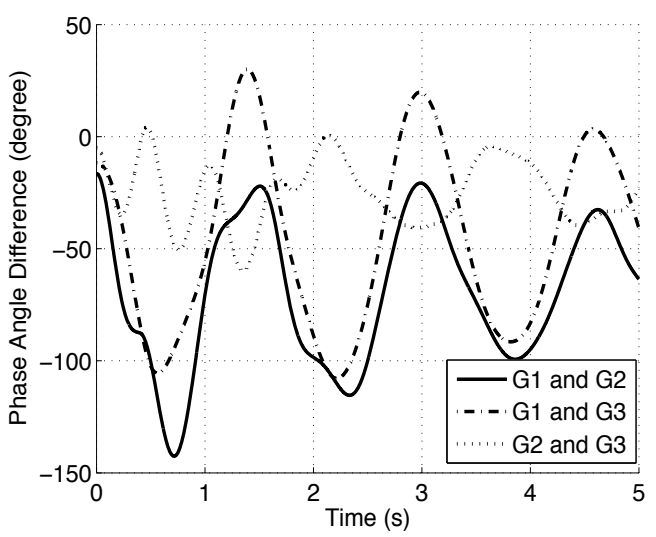

(c)

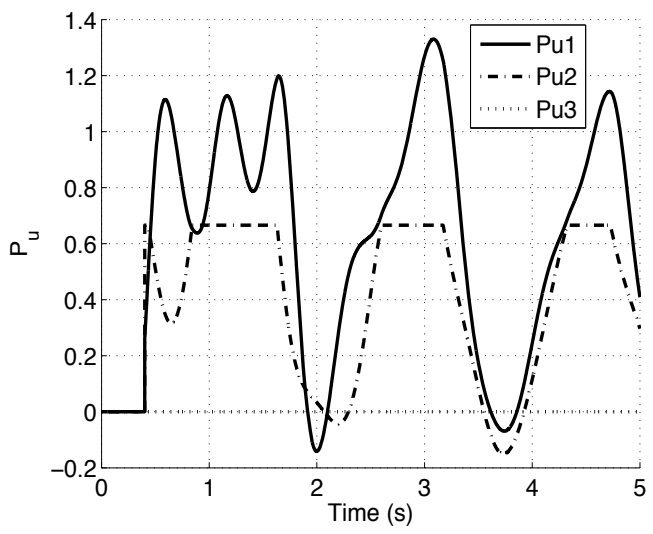

(d)

Fig. 6. Results of Two-Tier Control for Case II. 